\title{
Synthesis and structural characterization of new cadmium, zinc and iron based pyrophosphate: $(\mathrm{Cd}, \mathrm{Zn})_{2.5} \mathrm{Fe}\left(\mathrm{P}_{2} \mathrm{O}_{7}\right)_{2}$
}

\author{
Jamal Khmiyas, Abderrazzak Assani, Mohamed Saadi and Lahcen El Ammari. \\ Laboratoire de Chimie Appliquée des Matériaux, Centre des Sciences des Matériaux, Faculty of Sciences, \\ Mohammed V University in Rabat, Avenue Ibn Battouta, BP 1014, Rabat, Morocco.
}

\begin{abstract}
The novel compound with the following nominal formulation $(\mathrm{Cd}, \mathrm{Zn})_{2.5} \mathrm{Fe}\left(\mathrm{P}_{2} \mathrm{O}_{7}\right)_{2}$ has been successfully prepared by the solid state reaction and characterized by single-crystal X-ray diffraction. This phosphate crystallizes in the orthorhombic system with the $\mathrm{C} 222_{1}$ space group. Its crystal structure is formed by five types of cationic sites and by two unique $\left(\mathrm{P}_{2} \mathrm{O}_{7}\right)^{4-}$ anionic groups with a staggered conformation. In the structure, three sites are fulfilled by $\mathrm{Zn}^{2+}:(\mathrm{Zn}(3), \mathrm{Zn}(4))$ and $\mathrm{Fe}(1)^{3+}$, the fourth site is localized in the Wyckoff position $4 a$ (site symmetry $2 \ldots$ ) and is statically occupied by $\mathrm{Cd}^{2+}: \mathrm{Zn}^{2+}$ with ratio $0.586(2): 0.413(8)$. The last site, localized in $8 c$ Wyckoff position (site symmetry 1) is also occupied by a mixture of the two cations $\mathrm{Cd}^{2+}: \mathrm{Zn}^{2+}$ with occupancies of $0.444(6): 0.555(4)$. Within this framework, the metallic polyhedra $\left[M \mathrm{O}_{\mathrm{n}}\right](\mathrm{n}=5$ or 6$)$ form corrugated layers stacked along the $b$-axis, and intercalated by $\mathrm{P}_{2} \mathrm{O}_{7}$ groups.
\end{abstract}

\section{Introduction}

La chimie des matériaux phosphatés poreux et à charpente ouverte est en plein essor actuellement [1]. Ceci est dû essentiellement à la grande richesse et la diversité structurales et topologiques affichées par cette classe de matériaux. L'originalité des pyrophosphates tient, en majeure partie, à leurs diverses propriétés physicochimiques remarquables directement liées à leurs structures cristallines. Les pyrophosphates sont recherchées pour diverses applications potentielles à savoir : la catalyse [2], sorption \& désorption [3], autant que récepteur et capteur biologique [4], agent thérapeutique en hémodialyse [5], en agroalimentaire [6], conception de composite [7], biocéramique [8], inhibiteur endogène de la bio-minéralisation [9].

Dans ce cadre, on peut citer les pyrophosphates: $\mathrm{Na}_{3,12} \mathrm{Fe}_{2,44}\left(\mathrm{P}_{2} \mathrm{O}_{7}\right)_{2}$ [10], $\mathrm{Na}_{7} \mathrm{~V}_{3}\left(\mathrm{P}_{2} \mathrm{O}_{7}\right)_{4}$ [11] isotypes de $\mathrm{Na}_{7} \mathrm{M}_{3}\left(\mathrm{P}_{2} \mathrm{O}_{7}\right)_{4}(\mathrm{M}=\mathrm{Al}, \mathrm{Ga}, \mathrm{Cr}, \mathrm{Fe})$ [12] et $\mathrm{Na}_{2} \mathrm{MP}_{2} \mathrm{O}_{7}$ $(\mathrm{M}=\mathrm{Fe}, \mathrm{Mn}, \mathrm{Co})$ qui affichent d'excellents taux de capacités et de réversibilités ainsi qu'une grande stabilité thermique [13]. Par ailleurs, la famille des composés $\mathrm{A}^{\mathrm{I}} \mathrm{M}^{\mathrm{III}} \mathrm{P}_{2} \mathrm{O}_{7}(\mathrm{~A}=\mathrm{Li}, \mathrm{Na}, \mathrm{K}, \mathrm{Rb}, \mathrm{Cs}, \mathrm{Tl}, \mathrm{Ag})$ et $(\mathrm{M}=\mathrm{Al}$, $\mathrm{Ga}$, métal de transition), adoptent une structure à cage construite à partir des tétraèdres $\mathrm{PO}_{4}$ et $\mathrm{MO}_{6}$ partageant des sommets. Le site crée par cet agencement est occupé par des cations monovalents de coordinence $\mathrm{CN}=6$ [14].

L'élargissement des dimensions des espaces interstitiels, ainsi que l'occupation totale ou partielle des sites au sein de la structure cristalline est l'un des facteurs favorables à la mobilité des cations monovalents. De même, divers travaux ont été consacrés aux pyrophosphates métalliques et métalliques mixtes : $\mathrm{A}^{\mathrm{II}} \mathrm{B}^{\mathrm{II}} \mathrm{P}_{2} \mathrm{O}_{7}$ [15] $(\mathrm{A}=\mathrm{Ba}, \mathrm{Pb} ; \mathrm{B}=\mathrm{Pb}, \mathrm{Co}, \mathrm{Mn}, \mathrm{Cu})$, $\mathrm{A}^{\mathrm{II}} \mathrm{B}_{2}{ }^{\mathrm{III}}\left(\mathrm{P}_{2} \mathrm{O}_{7}\right)_{2}$ [16-18] $(\mathrm{A}=\mathrm{Sr}, \mathrm{Pb}, \mathrm{Cu} ; \mathrm{B}=\mathrm{Fe})$, et $\mathrm{A}^{\mathrm{I}} \mathrm{M}_{3}\left(\mathrm{P}_{2} \mathrm{O}_{7}\right)_{2} \quad(\mathrm{M}=\mathrm{Fe}, \mathrm{Co}, \mathrm{Ni})$ [19].Le présent travail relate la synthèse et la caractérisation structurale du pyrophosphate à base de cadmium, de zinc et de fer, $(\mathrm{Cd}, \mathrm{Zn})_{2.5} \mathrm{Fe}\left(\mathrm{P}_{2} \mathrm{O}_{7}\right)_{2}$

\section{Synthèse et cristallisation}

Les cristaux $\mathrm{du}$ nouveau pyrophosphate: $(\mathrm{Cd}, \mathrm{Zn})_{2.5} \mathrm{Fe}\left(\mathrm{P}_{2} \mathrm{O}_{7}\right)_{2}$ ont été synthétisés par réaction de diffusion à l'état solide, à partir des réactifs: $\mathrm{CdO}$ $(0,1284 \mathrm{~g}), \quad \mathrm{Zn}\left(\mathrm{NO}_{3}\right)_{2} \cdot 6 \mathrm{H}_{2} \mathrm{O} \quad(0,5949 \mathrm{~g}), \quad \mathrm{Fe}\left(\mathrm{NO}_{3}\right)_{3} \cdot 9 \mathrm{H}_{2} \mathrm{O}$ $(0,4040 \mathrm{~g})$ et de $\mathrm{H}_{3} \mathrm{PO}_{4}(85 \%)(0.20 \mathrm{~mL})$, selon les rapports $\mathrm{Cd}: \mathrm{Zn}: \mathrm{Fe}: \mathrm{P}=1: 2: 1: 3$. Dans un bécher contenant de l'eau distillée, le mélange réactionnel est homogénéisé par agitation à l'ambiante pendant 24 heures. Après une évaporation lente, une poudre homogène de couleur jaune est obtenue. Cette poudre est ensuite broyée pendant 30 minutes dans un mortier en agate, puis mis dans un creuset en platine et puis porté lentement à la fusion à $1218 \mathrm{~K}$ et maintenu à cette température pendant 4 heures.

Après un refroidissement lent avec une vitesse de $5^{\circ} \mathrm{C} / \mathrm{h}$ et un lavage à l'éthanol, dans un bain à ultrasons, nous avons obtenus des cristaux marron relatifs à cette nouvelle phase. 


\section{Etude structurale du nouveau pyro- phosphate : $(\mathrm{Cd}, \mathrm{Zn})_{2.5} \mathrm{Fe}\left(\mathrm{P}_{2} \mathrm{O}_{7}\right)_{2}$}

\section{1 collecte des intensités diffractées}

Un cristal marron de bonne qualité et de taille exploitable, a été sélectionné et monté sur le diffractomètre à 4 cercles X8 APEXII de l'UATRS (Unités d'Appui Technique à la Recherche Scientifique) du CNRST (Centre National pour la Recherche Scientifique et Technique). La collecte des intensités de diffraction des rayons-X, a été effectuée dans les conditions mentionnées dans le tableau(1).

\subsection{Résolution et affinement structural}

La structure a été résolue par la méthode de Patterson à l'aide du programme SHLEXS-97 et affinée par la méthode des moindres carrées à l'aide du programme SHELXL-97 [20]. La résolution nous a permis de localiser les atomes lourds $\mathrm{Cd}, \mathrm{Zn}, \mathrm{Fe}$ et $\mathrm{P}$. Suite aux séries d'affinements successifs suivies de Fourierdifférences, nous avons pu localiser les quatorze atomes d'oxygène indépendants dans l'unité asymétrique. La structure est caractérisée par désordre partiel.

En effet le dernier cycle d'affinement conduit aux résultats suivants: les atomes $\mathrm{Cd}(1)$ et $\mathrm{Zn}(1)$ sont réparties statiquement sur la position particulière (4a) avec un taux d'occupation de 0,586(6) et 0,414(6) respectivement. De même $\operatorname{Cd}(2)$ et $\operatorname{Zn}(2)$ occupent la même position générale (8c) avec le taux de $0.445(5)$ et $0.555(5)$ respectivement. Le reste des atomes occupent totalement leur site cristallographique.

Cette dernière répartition conduit à la formulation suivante : $\mathrm{Cd}_{0.74} \mathrm{Zn}_{1.76} \mathrm{Fe}\left(\mathrm{P}_{2} \mathrm{O}_{7}\right)_{2}$. L'affinement structural a abouti à de bons facteurs de reliabilité consignés dans le tableau (1). Les coordonnées réduites et les déplacements atomiques isotropes et anisotropes sont rassemblés dans les tableaux (2) et (3), respectivement.

Tableau 1. Données cristallographiques et conditions d'enregistrement des intensités diffractées du nouveau pyrophosphate $\mathrm{Cd}_{0.74} \mathrm{Zn}_{1.76} \mathrm{Fe}\left(\mathrm{P}_{2} \mathrm{O}_{7}\right)_{2}$.

\begin{tabular}{|l|c|}
\hline \multicolumn{2}{|c|}{ Données cristallographiques } \\
\hline Formule chimique & $\mathrm{Cd}_{0.74} \mathrm{Zn}_{1.76} \mathrm{Fe}\left(\mathrm{P}_{2} \mathrm{O}_{7}\right)_{2}$ \\
\hline Système cristallin & Orthorhombique \\
\hline Groupe d'espace & $\mathrm{C} 222_{1}$ \\
\hline Paramètres de la maille & $\begin{array}{l}a=8,4761(1) \AA \\
\mathrm{b}=9,7581(1) \AA \\
\mathrm{c}=24,0557(3) \AA\end{array}$ \\
\hline Volume de la maille $\left(\AA^{3}\right)$ & $1989,66(4)$ \\
\hline Nombre de motif par maille $\mathrm{Z}$ & 8 \\
\hline Densité calculée $\left(\mathrm{Mg} \cdot \mathrm{m}^{-3}\right)$ & 4.018 \\
\hline Coefficient d'absorption $\left(\mathrm{mm}^{-1}\right)$ & 7.934 \\
\hline
\end{tabular}

\begin{tabular}{|c|c|}
\hline \multicolumn{2}{|c|}{ Collecte des données et résultats d'affinement } \\
\hline Diffractomètre & Bruker X8 APEXII \\
\hline Température $(\mathrm{K})$ & 296 \\
\hline Longueur d'onde $\mathrm{Mo}(\mathrm{K} \alpha)(\AA)$ & 0,7071 \\
\hline Correction d'absorption & $\begin{array}{c}\text { multi-scan } \\
\text { (SADABS; Bruker, } \\
\text { 2005) }\end{array}$ \\
\hline Domaine de $\theta\left(^{\circ}\right)$ & $\begin{array}{c}\theta_{\max }=36.6 \\
\theta_{\min }=3.2\end{array}$ \\
\hline Indices de Miller & $\begin{aligned} h & =-14 \rightarrow 14 \\
k & =-16 \rightarrow 16 \\
l & =-40 \rightarrow 35\end{aligned}$ \\
\hline Réflexions mesurées & 16814 \\
\hline Réflexions indépendantes & 4898 \\
\hline Réflexions pour $\mathrm{I}>2 \sigma(\mathrm{I})$ & 4796 \\
\hline $\mathrm{R}_{\mathrm{int}}$ & 0.021 \\
\hline$R\left[F^{2}>2 \sigma\left(F^{2}\right)\right]$ & 0.020 \\
\hline$w R\left(F^{2}\right)$ & 0.051 \\
\hline$S$ & 1.09 \\
\hline Nombre des paramètres affinés & 199 \\
\hline
\end{tabular}

\subsection{Description de la structure}

La structure du nouveau pyrophosphate de cadmium, zinc et de fer $\mathrm{Cd}_{0.74} \mathrm{Zn}_{1.76} \mathrm{Fe}\left(\mathrm{P}_{2} \mathrm{O}_{7}\right)_{2}$ est isotype de : $\mathrm{Fe}_{5}{ }_{5} \mathrm{Fe}_{2} \mathrm{III}_{2}\left(\mathrm{P}_{2} \mathrm{O}_{7}\right)_{4}[21], \mathrm{Cd}_{5} \mathrm{MIII}_{2}\left(\mathrm{P}_{2} \mathrm{O}_{7}\right)_{4}(\mathrm{M}=\mathrm{V}, \mathrm{Fe})[22]$ et $\mathrm{Cd}_{4.12} \mathrm{FeII}_{0.88} \mathrm{FeIII}\left(\mathrm{P}_{2} \mathrm{O}_{7}\right)_{4} \quad[23]$. Les principales distances et angles interatomiques de la structure de $\mathrm{Cd}_{0.74} \mathrm{Zn}_{1.76} \mathrm{Fe}\left(\mathrm{P}_{2} \mathrm{O}_{7}\right)_{2}$ sont reportés dans le tableau (4).

Dans la structure de ce nouveau pyrophosphate, la séquence de polyèdres $(\mathrm{Cd}(1) / \mathrm{Zn}(1)) \mathrm{O}_{6} \quad-\mathrm{FeO}_{6}$ $(\mathrm{Cd}(2) / \mathrm{Zn}(2)) \mathrm{O}_{5}-\mathrm{FeO}_{6}-(\mathrm{Cd}(2) / \mathrm{Zn}(2)) \mathrm{O}_{5}-\mathrm{Zn}(4) \mathrm{O}_{6}$ permet de construire un anneau à six polyèdres (Figure 1-a). En effet, les cinq premiers polyèdres de cette succession sont liés entre eux par la mise en commun d'une arête, l'octaèdre $\mathrm{Zn}(4) \mathrm{O}_{6}$ partage deux de ces oxygènes avec $(\mathrm{Cd}(1) / \mathrm{Zn}(1)) \mathrm{O}_{6}$ et $(\mathrm{Cd}(2) / \mathrm{Zn}(2)) \mathrm{O}_{5}$. De même, chacun des anneaux partage une arête de l'octaèdre $\mathrm{Zn}(4) \mathrm{O}_{6}$ avec l'octaèdre $(\mathrm{Cd}(1) / \mathrm{Zn}(1)) \mathrm{O}_{6}$ de l'anneau adjacent, pour engendrer des chaînes d'anneaux infinies évoluant le long de l'axe- $a$ à $\mathrm{z} \approx 0$ et à $\mathrm{z} \approx 1 / 2$ (Figure 1-b).

Les octaèdres $\mathrm{Zn}(3) \mathrm{O}_{6}$, qui ne contribuent pas à la formation des anneaux, viennent relier ces deux types de chaînes entre elles pour former des feuillets $\left[(\mathrm{Cd}, \mathrm{Zn})_{3} \mathrm{Zn}_{2} \mathrm{Fe}_{2} \mathrm{O}_{24}\right]_{\infty}$ ondulés parallèles au plan $(010)$ (Figure $2 \mathrm{a}$ et $2 \mathrm{~b}$ ).Les deux types de groupements $\mathrm{P}_{2} \mathrm{O}_{7}$ sont isolés (Figure 3 ) et sont disposés de manière ondulée suivant l'axe c . Les feuillets $\left[(\mathrm{Cd}, \mathrm{Zn})_{3} \mathrm{Zn}_{2} \mathrm{Fe}_{2} \mathrm{O}_{24}\right]_{\infty}$ sont inter-connectés par les groupements $\mathrm{P}_{2} \mathrm{O}_{7}$ pour aboutir à un réseau tridimensionnel (Figure 4).

Signalons que les groupements $\mathrm{P}_{2} \mathrm{O}_{7}$ adoptent une conformation décalée, également observée dans l'homologue : $\mathrm{Co}_{5} \mathrm{~V}^{\mathrm{III}}{ }_{2}\left(\mathrm{P}_{2} \mathrm{O}_{7}\right)_{4}$ [24]. 
Tableau 2. Coordonnées atomiques et facteurs de déplacements atomiques isotropes équivalents $\left(\AA^{2}\right)$

\begin{tabular}{|c|c|c|c|c|c|c|}
\hline Atome & Site cristallographique & $\mathbf{x}$ & $\mathbf{y}$ & $\mathbf{z}$ & Uiso*/Ueq & Occupation \\
\hline Cd1 & $4 a$ & $0.19839(4)$ & 0.5 & 0.5 & $0.0104(1)$ & $0,586(6)$ \\
\hline Zn1 & $4 a$ & $0.19839(4)$ & 0.5 & 0.5 & $0.0104(1)$ & $0,414(6)$ \\
\hline Cd2 & $8 c$ & $0.31562(3)$ & $0.64219(3)$ & $0.86008(2)$ & $0.01278(9)$ & $0,445(5)$ \\
\hline Zn2 & $8 c$ & $0.31562(3)$ & $0.64219(3)$ & $0.86008(2)$ & $0.01278(9)$ & $0,555(5)$ \\
\hline Zn3 & $4 b$ & 0 & $0.29054(5)$ & 0.75 & $0.00867(9)$ & 1 \\
\hline Zn4 & $4 a$ & $0.08425(6)$ & 1 & 0.5 & $0.0225(1)$ & 1 \\
\hline Fe1 & $8 c$ & $0.07277(4)$ & $0.65109(4)$ & $0.62175(2)$ & $0.00626(7)$ & 1 \\
\hline P1 & $8 c$ & $0.40301(8)$ & $0.79463(7)$ & $0.49719(3)$ & $0.0077(1)$ & 1 \\
\hline P2 & $8 c$ & $0.24181(8)$ & $0.54279(7)$ & $0.73124(3)$ & $0.0075(1)$ & 1 \\
\hline P3 & $8 c$ & $0.37948(8)$ & $0.44633(7)$ & $0.62565(3)$ & $0.0070(1)$ & 1 \\
\hline P4 & $8 c$ & $0.39000(8)$ & $0.85839(7)$ & $0.61800(3)$ & $0.0070(1)$ & 1 \\
\hline O1 & $8 c$ & $0.3317(3)$ & $0.6361(2)$ & $0.77110(9)$ & $0.0133(4)$ & 1 \\
\hline O2 & $8 c$ & $0.3839(2)$ & $1.0141(2)$ & $0.62598(9)$ & $0.0099(3)$ & 1 \\
\hline O3 & $8 c$ & $0.2267(2)$ & $0.4950(2)$ & $0.59825(8)$ & $0.0106(3)$ & 1 \\
\hline O4 & $8 c$ & $0.3991(3)$ & $0.6393(2)$ & $0.49590(9)$ & $0.0127(3)$ & 1 \\
\hline O5 & $8 c$ & $0.3867(3)$ & $0.2886(2)$ & $0.62753(9)$ & $0.0114(3)$ & 1 \\
\hline O6 & $8 c$ & $0.2298(3)$ & $0.7948(2)$ & $0.6163(1)$ & $0.0140(4)$ & 1 \\
\hline O7 & $8 c$ & $0.5060(3)$ & $0.7911(2)$ & $0.6573(1)$ & $0.0149(4)$ & 1 \\
\hline O8 & $8 c$ & $0.1817(3)$ & $0.4171(2)$ & $0.7593(1)$ & $0.0169(4)$ & 1 \\
\hline O9 & $8 c$ & $0.5384(2)$ & $0.8524(2)$ & $0.46189(9)$ & $0.0119(3)$ & 1 \\
\hline O10 & $8 c$ & $0.1177(3)$ & $0.6188(2)$ & $0.69813(9)$ & $0.0129(4)$ & 1 \\
\hline O11 & $8 c$ & $0.5240(2)$ & $0.5101(2)$ & $0.5995(1)$ & $0.0138(4)$ & 1 \\
\hline O12 & $8 c$ & $0.4653(3)$ & $0.8385(3)$ & $0.5573(1)$ & $0.0168(4)$ & 1 \\
\hline O13 & $8 c$ & $0.2462(3)$ & $0.8551(3)$ & $0.4830(1)$ & $0.0201(5)$ & 1 \\
\hline O14 & $8 c$ & $0.3786(2)$ & $0.4973(2)$ & $0.68812(9)$ & $0.0124(3)$ & 1 \\
\hline
\end{tabular}

Tableau 3. Facteurs de déplacements atomiques anisotropes $\left(\AA^{2}\right)$.

\begin{tabular}{|c|c|c|c|c|c|c|}
\hline & $\mathrm{U} 11$ & $\mathrm{U} 22$ & $\mathrm{U} 33$ & $\mathrm{U} 12$ & $\mathrm{U} 13$ & $\mathrm{U} 23$ \\
\hline $\mathrm{Cd} 1 / \mathrm{Zn} 1$ & $0.0083(1)$ & $0.0087(1)$ & $0.0143(1)$ & 0.000 & 0.000 & $-0.0029(1)$ \\
\hline $\mathrm{Cd}$ /Zn2 & $0.0072(1)$ & $0.0115(1)$ & $0.0197(1)$ & $-0.00155(8)$ & $0.00004(8)$ & $0.00535(9)$ \\
\hline Zn3 & $0.0086(1)$ & $0.0076(1)$ & $0.0099(1)$ & 0.000 & $0.0008(1)$ & 0.000 \\
\hline Zn4 & $0.0041(1)$ & $0.0057(1)$ & $0.0582(4)$ & 0.000 & 0.000 & $-0.0059(2)$ \\
\hline Fe1 & $0.0069(1)$ & $0.0055(1)$ & $0.0065(1)$ & $-0.0005(1)$ & $-0.0004(1)$ & $0.0007(1)$ \\
\hline P1 & $0.0087(2)$ & $0.0054(2)$ & $0.0089(2)$ & $-0.0009(1)$ & $0.0012(2)$ & $0.0005(2)$ \\
\hline P2 & $0.0090(3)$ & $0.0069(2)$ & $0.0064(2)$ & $-0.0010(2)$ & $-0.0011(1)$ & $-0.0008(2)$ \\
\hline P3 & $0.0079(2)$ & $0.0055(2)$ & $0.0076(3)$ & $0.0015(1)$ & $0.0010(1)$ & $0.0006(2)$ \\
\hline P4 & $0.0087(2)$ & $0.0051(2)$ & $0.0073(2)$ & $-0.0011(2)$ & $-0.0009(1)$ & $-0.0001(2)$ \\
\hline O1 & $0.0153(9)$ & $0.0131(8)$ & $0.0116(8)$ & $-0.0007(8)$ & $-0.0041(7)$ & $-0.0061(7)$ \\
\hline O2 & $0.0149(8)$ & $0.0040(7)$ & $0.0108(8)$ & $-0.0007(6)$ & $0.0010(6)$ & $0.0008(6)$ \\
\hline O3 & $0.0097(7)$ & $0.0125(8)$ & $0.0096(8)$ & $0.0021(7)$ & $-0.0016(6)$ & $0.0015(7)$ \\
\hline O4 & $0.0202(9)$ & $0.0062(7)$ & $0.0118(8)$ & $-0.0016(7)$ & $-0.0026(8)$ & $0.0008(7)$ \\
\hline O5 & $0.0160(9)$ & $0.0056(7)$ & $0.0126(9)$ & $0.0018(6)$ & $0.0010(7)$ & $0.0012(6)$ \\
\hline O6 & $0.0106(8)$ & $0.0109(8)$ & $0.021(1)$ & $-0.0044(7)$ & $0.0003(7)$ & $-0.0011(8)$ \\
\hline O7 & $0.0169(9)$ & $0.0108(8)$ & $0.0170(9)$ & $0.0032(7)$ & $-0.0062(8)$ & $0.0023(7)$ \\
\hline O8 & $0.0225(1)$ & $0.0127(9)$ & $0.016(1)$ & $-0.0042(8)$ & $0.0020(8)$ & $0.0045(7)$ \\
\hline O9 & $0.0109(7)$ & $0.0151(8)$ & $0.0097(8)$ & $-0.0008(7)$ & $0.0031(6)$ & $0.0047(7)$ \\
\hline O10 & $0.0117(8)$ & $0.0184(9)$ & $0.0086(8)$ & $0.0038(7)$ & $-0.0020(6)$ & $-0.0002(7)$ \\
\hline O11 & $0.0110(8)$ & $0.0134(8)$ & $0.0170(9)$ & $0.0004(7)$ & $0.0058(7)$ & $0.0040(8)$ \\
\hline O12 & $0.0148(9)$ & $0.0223(1)$ & $0.0132(9)$ & $-0.0062(8)$ & $0.0035(7)$ & $-0.0095(8)$ \\
\hline O13 & $0.0101(8)$ & $0.0175(1)$ & $0.033(1)$ & $0.0010(8)$ & $-0.0003(8)$ & $0.005(1)$ \\
\hline O14 & $0.0102(7)$ & $0.0168(8)$ & $0.0102(8)$ & $0.0017(7)$ & $-0.0009(6)$ & $-0.0032(8)$ \\
\hline & & & & & & \\
\hline
\end{tabular}




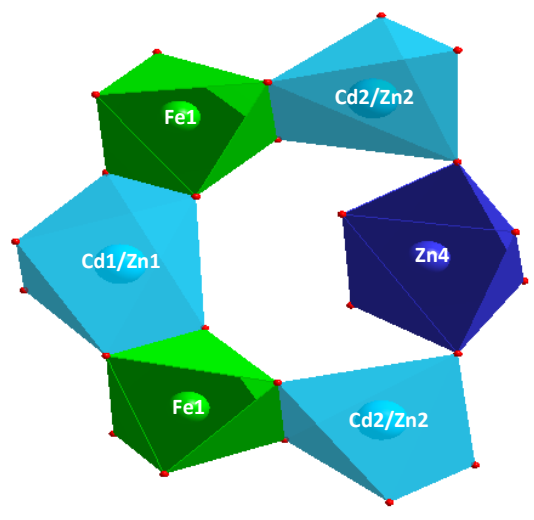

(a)

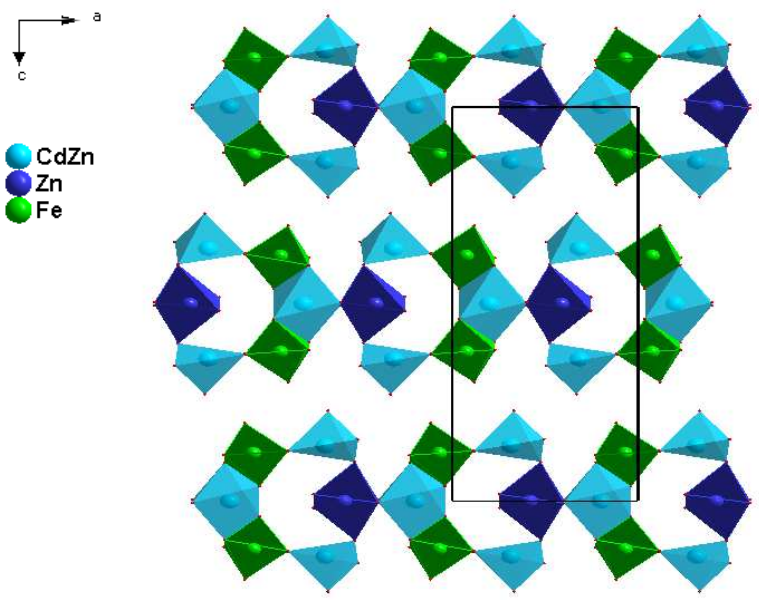

(b)

Fig.1. (a) : Alternance des octaèdres $\mathrm{MO}_{6}\left(\mathrm{M}=\mathrm{Fe}^{\mathrm{III}}, \mathrm{Zn}\right.$, $(\mathrm{Cd}(1), \mathrm{Zn}(1))$ et la pyramide à base carrée $(\mathrm{Cd}(2) / \mathrm{Zn}(2)) \mathrm{O}_{5}$ formant l'anneau à 6 polyèdres. (b) : Succession des chaînes d'anneaux dans le plan (001).

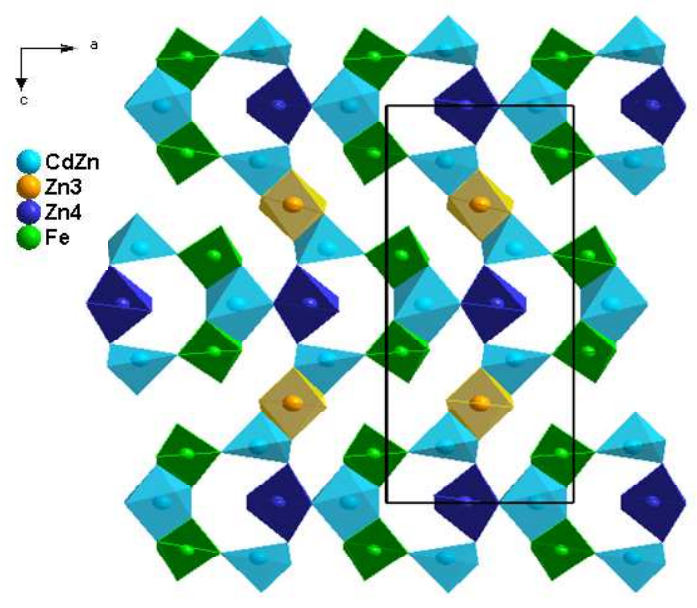

(a)

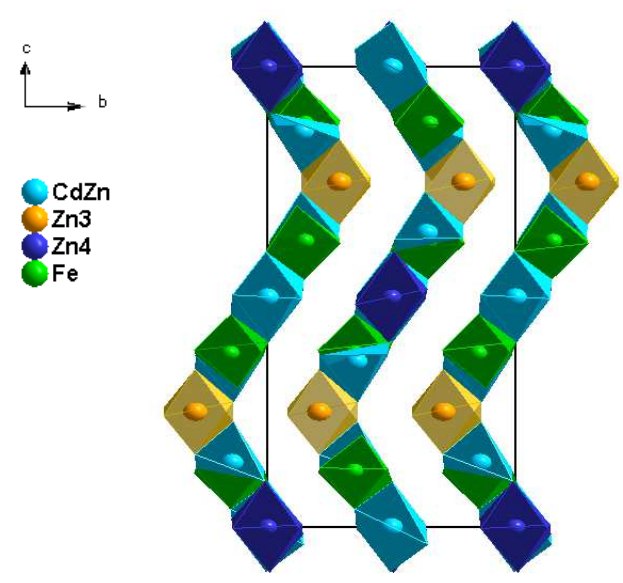

(b)

Fig.2 (a) : Projection d'une couche disposée parallèlement au plan (010).(b) : Projection des couches dans le plan (b, c).

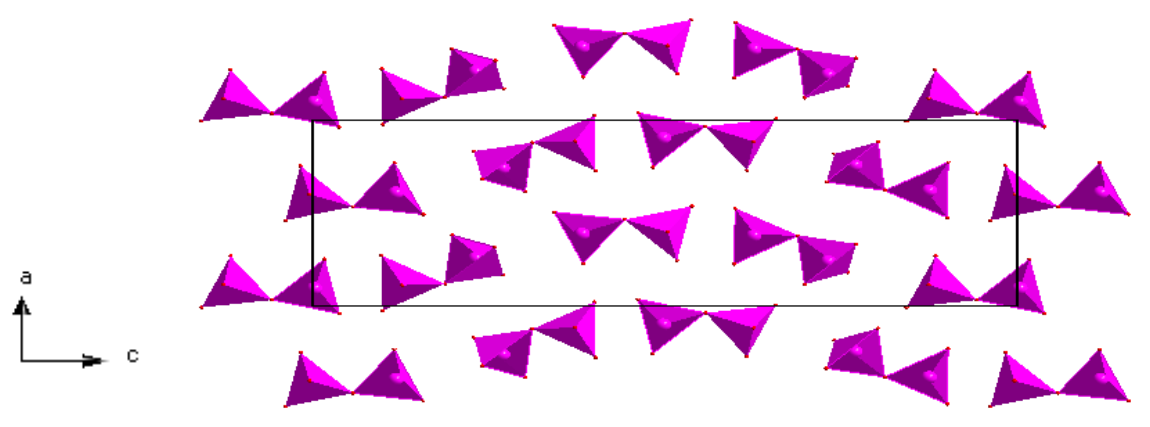

Fig.3 : Agencement des groupements $\mathrm{P}_{2} \mathrm{O}_{7}$ reparti suivant l'axe b. 


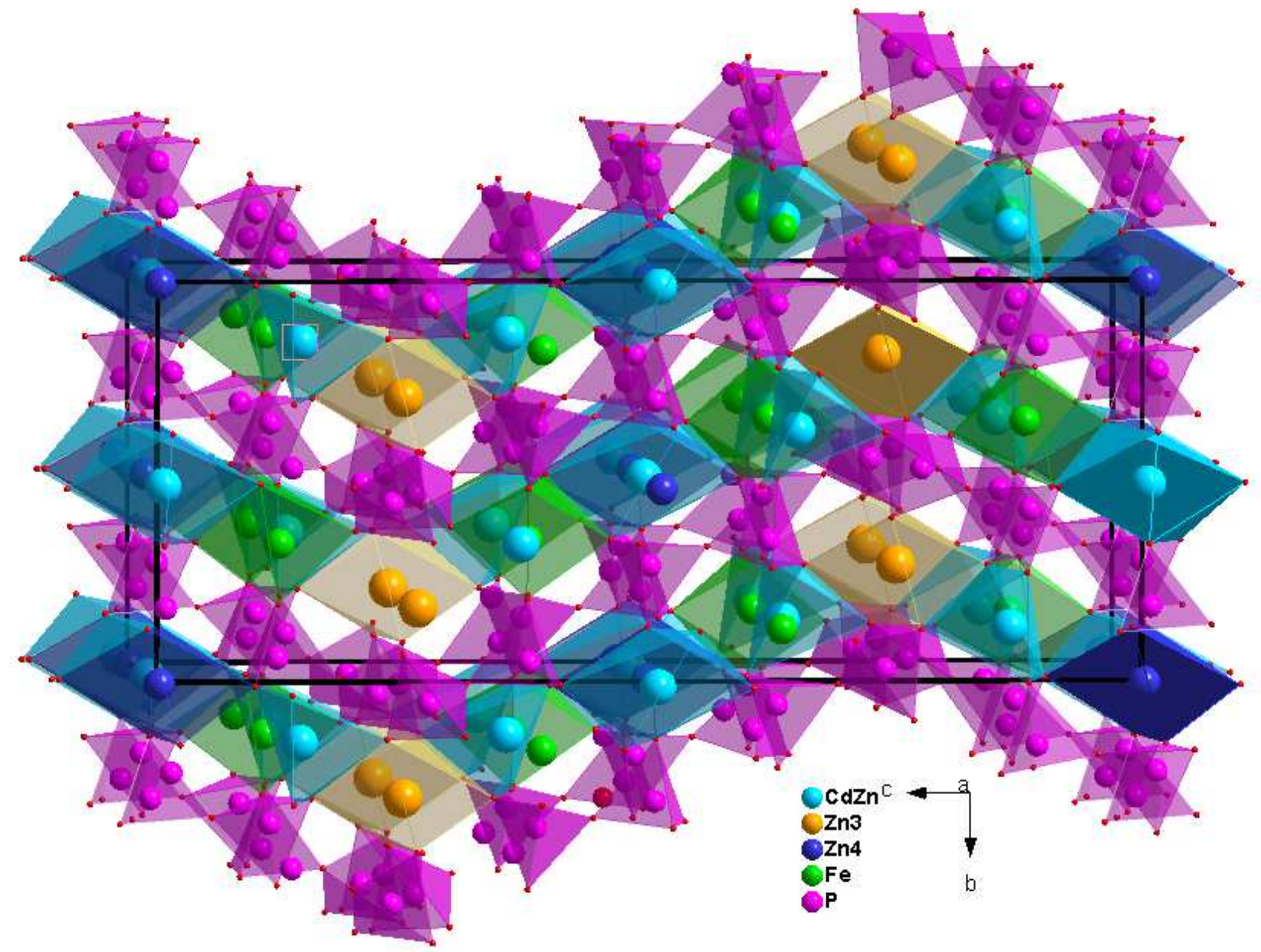

Fig.4.Structure tridimensionnelle du nouveau pyrophosphate $\mathrm{Cd}_{0.74} \mathrm{Zn}_{1.76} \mathrm{Fe}\left(\mathrm{P}_{2} \mathrm{O}_{7}\right)_{2}$.

Tableau 4. Distances $(\AA \dot{)})$ et angles interatomiques $\left(^{\circ}\right)$ pour le pyrophosphate $\mathrm{Cd}_{0.74} \mathrm{Zn}_{1.76} \mathrm{Fe}\left(\mathrm{P}_{2} \mathrm{O}_{7}\right)_{2}$.

\begin{tabular}{|c|c|}
\hline \multicolumn{2}{|c|}{ Distances Fe(1) - O (§̊) } \\
\hline $\mathrm{Fe} 1-\mathrm{O} 10$ & $1.903(2)$ \\
\hline $\mathrm{Fe} 1-\mathrm{O} 6$ & $1.938(2)$ \\
\hline $\mathrm{Fe} 1-\mathrm{O} 9^{\text {iii }}$ & $2.033(2)$ \\
\hline $\mathrm{Fe} 1-\mathrm{O} 5^{\mathrm{x}}$ & $2.075(2)$ \\
\hline $\mathrm{Fe} 1-\mathrm{O} 3$ & $2.084(2)$ \\
\hline $\mathrm{Fe} 1-\mathrm{O} 2^{\mathrm{ii}}$ & $2.088(2)$ \\
\hline Distance moyenne $<\mathrm{Fe}(1)-\mathrm{O}>$ & 2.020 \\
\hline \multicolumn{2}{|c|}{ Angles O - Fe(1) - O ( $\left.{ }^{\circ}\right)$} \\
\hline $\mathrm{O} 6-\mathrm{Fe} 1-\mathrm{O} 9^{\text {iii }}$ & $92.5(1)$ \\
\hline $\mathrm{O} 10-\mathrm{Fe} 1-\mathrm{O}^{\mathrm{x}}$ & $101.20(9)$ \\
\hline $\mathrm{O} 6-\mathrm{Fe} 1-\mathrm{O}^{\mathrm{x}}$ & $93.36(9)$ \\
\hline $\mathrm{O}^{\mathrm{iii}}-\mathrm{Fe} 1-\mathrm{O}^{\mathrm{x}}$ & $88.17(9)$ \\
\hline $\mathrm{O} 10-\mathrm{Fe} 1-\mathrm{O} 3$ & $90.90(9)$ \\
\hline $\mathrm{O} 6-\mathrm{Fe} 1-\mathrm{O} 3$ & $94.61(9)$ \\
\hline $\mathrm{O} 9^{\mathrm{iii}}-\mathrm{Fe} 1-\mathrm{O} 3$ & $79.00(8)$ \\
\hline $\mathrm{O} 10-\mathrm{Fe} 1-\mathrm{O} 2^{\mathrm{ii}}$ & $90.02(9)$ \\
\hline $\mathrm{O} 9^{\mathrm{iii}}-\mathrm{Fe} 1-\mathrm{O} 2^{\mathrm{ii}}$ & $85.85(9)$ \\
\hline $\mathrm{O} 5^{\mathrm{x}}-\mathrm{Fe} 1-\mathrm{O} 2^{\mathrm{ii}}$ & $80.10(8)$ \\
\hline $\mathrm{O} 3-\mathrm{Fe} 1-\mathrm{O} 2^{\mathrm{ii}}$ & $91.46(8)$ \\
\hline $\mathrm{O} 10-\mathrm{Fe} 1-\mathrm{O} 6$ & $92.7(1)$ \\
\hline $\mathrm{O} 6-\mathrm{Fe} 1-\mathrm{O} 2^{\mathrm{ii}}$ & $173.29(9)$ \\
\hline \multicolumn{2}{|c|}{ Distances Cd(1)/Zn(1) - O (§) } \\
\hline
\end{tabular}

\begin{tabular}{|c|c|}
\hline $\mathrm{Cd} 1 / \mathrm{Zn} 1-\mathrm{O} 4$ & $2.179(2)$ \\
\hline $\mathrm{Cd} 1 / \mathrm{Zn} 1-\mathrm{O} 4^{\mathrm{i}}$ & $2.179(2)$ \\
\hline $\mathrm{Cd} 1 / \mathrm{Zn} 1-\mathrm{O}^{\mathrm{ii}}$ & $2.181(2)$ \\
\hline $\mathrm{Cd} 1 / \mathrm{Zn} 1-\mathrm{O9}^{\mathrm{iii}}$ & $2.181(2)$ \\
\hline $\mathrm{Cd} 1 / \mathrm{Zn} 1-\mathrm{O}^{\mathrm{i}}$ & $2.376(2)$ \\
\hline $\mathrm{Cd} 1 / \mathrm{Zn} 1-\mathrm{O} 3$ & $2.376(2)$ \\
\hline Distance moyenne $<\mathrm{Cd}(1) / \mathrm{Zn}(1)-\mathrm{O}>$ & 2.245 \\
\hline \multicolumn{2}{|c|}{ Angles $\mathrm{O}-\mathrm{Cd}(1) / \mathrm{Zn}(1)-\mathrm{O}\left(^{\circ}\right)$} \\
\hline $\mathrm{O} 4-\mathrm{Cd} 1 / \mathrm{Zn} 1-\mathrm{O}^{\mathrm{i}}$ & $77.4(1)$ \\
\hline $\mathrm{O} 4^{\mathrm{i}}-\mathrm{Cd} 1 / \mathrm{Zn} 1-\mathrm{O} 9^{\text {ii }}$ & $95.32(8)$ \\
\hline $\mathrm{O} 4-\mathrm{Cd} 1 / \mathrm{Zn} 1-\mathrm{O}^{\mathrm{iii}}$ & $95.32(8)$ \\
\hline $\mathrm{O}^{\mathrm{ii}}-\mathrm{Cd} 1 / \mathrm{Zn} 1-\mathrm{O}^{\mathrm{iii}}$ & $103.1(1)$ \\
\hline $\mathrm{O} 4-\mathrm{Cd} 1 / \mathrm{Zn} 1-\mathrm{O}^{\mathrm{i}}$ & $82.14(8)$ \\
\hline $\mathrm{O} 4-\mathrm{Cd} 1 / \mathrm{Zn} 1-\mathrm{O} 3^{\mathrm{i}}$ & $88.79(8)$ \\
\hline $\mathrm{O} 9^{\mathrm{ii}}-\mathrm{Cd} 1 / \mathrm{Zn} 1-\mathrm{O} 3^{\mathrm{i}}$ & $70.02(7)$ \\
\hline $\mathrm{O} 9^{\text {iii }}-\mathrm{Cd} 1 / \mathrm{Zn} 1-\mathrm{O}^{\mathrm{i}}$ & $117.87(7)$ \\
\hline $\mathrm{O} 4-\mathrm{Cd} 1 / \mathrm{Zn} 1-\mathrm{O} 3$ & $88.79(8)$ \\
\hline $\mathrm{O} 4{ }^{\mathrm{i}}-\mathrm{Cd} 1 / \mathrm{Zn} 1-\mathrm{O} 3$ & $82.14(8)$ \\
\hline $\mathrm{O}^{\mathrm{ii}}-\mathrm{Cd} 1 / \mathrm{Zn} 1-\mathrm{O} 3$ & $117.87(8)$ \\
\hline $\mathrm{O} 9^{\text {iii }}-\mathrm{Cd} 1 / \mathrm{Zn} 1-\mathrm{O} 3$ & $70.02(7)$ \\
\hline $\mathrm{O} 3{ }^{\mathrm{i}}-\mathrm{Cd} 1 / \mathrm{Zn} 1-\mathrm{O} 3$ & $168.40(10)$ \\
\hline \multicolumn{2}{|c|}{ Distances Cd(2)/Zn(2) - O (A) } \\
\hline $\mathrm{Cd} 2 / \mathrm{Zn} 2-\mathrm{O} 11^{\mathrm{v}}$ & $2.110(2)$ \\
\hline $\mathrm{Cd} 2 / \mathrm{Zn} 2-\mathrm{O} 2^{\mathrm{vi}}$ & $2.130(2)$ \\
\hline $\mathrm{Cd} 2 / \mathrm{Zn} 2-\mathrm{O}^{\mathrm{v}}$ & $2.138(2)$ \\
\hline
\end{tabular}




\begin{tabular}{|c|c|}
\hline $\mathrm{Cd} 2 / \mathrm{Zn} 2-\mathrm{O} 1$ & $2.146(2)$ \\
\hline $\mathrm{Cd} 2 / \mathrm{Zn} 2-\mathrm{O}^{\text {vii }}$ & $2.252(2)$ \\
\hline Distance moyenne $<\mathrm{Cd}(2) / \mathrm{Zn}(2)-\mathrm{O}>$ & 2.1552 \\
\hline \multicolumn{2}{|c|}{ Angles $\mathrm{O}-\mathrm{Cd}(2) / \mathrm{Zn}(2)-\mathrm{O}\left(^{\circ}\right)$} \\
\hline $\mathrm{O} 11^{\mathrm{v}}-\mathrm{Cd} 2 / \mathrm{Zn} 2-\mathrm{O} 2^{\mathrm{vi}}$ & $94.64(8)$ \\
\hline $\mathrm{O} 11^{\mathrm{v}}-\mathrm{Cd} 2 / \mathrm{Zn} 2-\mathrm{O}^{\mathrm{v}}$ & $92.85(9)$ \\
\hline $\mathrm{O} 11^{\mathrm{v}}-\mathrm{Cd} 2 / \mathrm{Zn} 2-\mathrm{O} 1$ & $113.66(8)$ \\
\hline $\mathrm{O} 2^{\mathrm{vi}}-\mathrm{Cd} 2 / \mathrm{Zn} 2-\mathrm{O} 1$ & $101.03(8)$ \\
\hline $\mathrm{O} 7^{\mathrm{V}}-\mathrm{Cd} 2 / \mathrm{Zn} 2-\mathrm{O} 1$ & $77.22(9)$ \\
\hline $\mathrm{O} 2^{\mathrm{vi}}-\mathrm{Cd} 2 / \mathrm{Zn} 2-\mathrm{O}^{\text {vii }}$ & $75.32(7)$ \\
\hline $\mathrm{O} 7^{\mathrm{v}}-\mathrm{Cd} 2 / \mathrm{Zn} 2-\mathrm{O}^{\mathrm{vii}}$ & $97.65(8)$ \\
\hline $\mathrm{O} 1-\mathrm{Cd} 2 / \mathrm{Zn} 2-\mathrm{O}^{\mathrm{vii}}$ & $101.42(8)$ \\
\hline \multicolumn{2}{|l|}{ Distances $\mathrm{Zn}(3)-\mathrm{O}(\AA)$} \\
\hline $\mathrm{Zn} 3-\mathrm{O}^{\text {viii }}$ & $1.987(2)$ \\
\hline $\mathrm{Zn} 3-\mathrm{O} 8$ & $1.987(2)$ \\
\hline $\mathrm{Zn} 3-\mathrm{O} 1^{\mathrm{ii}}$ & $2.136(2)$ \\
\hline $\mathrm{Zn} 3-\mathrm{O} 1^{\mathrm{vi}}$ & $2.136(2)$ \\
\hline $\mathrm{Zn} 3-\mathrm{O}^{\mathrm{ii}}$ & $2.230(2)$ \\
\hline $\mathrm{Zn} 3-\mathrm{O}^{\mathrm{vi}}$ & $2.230(2)$ \\
\hline Distance moyenne $<\mathrm{Zn}(3)-\mathrm{O}>$ & 2.117 \\
\hline \multicolumn{2}{|l|}{ Angles O - Zn(3) - O $\left(^{\circ}\right)$} \\
\hline $\mathrm{O} 8^{\text {viii }-\mathrm{Zn} 3-\mathrm{O} 8}$ & $103.2(1)$ \\
\hline 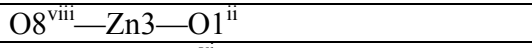 & $87.0(1)$ \\
\hline $\mathrm{O} 8-\mathrm{Zn} 3-\mathrm{O} 1^{\mathrm{vi}}$ & $87.0(1)$ \\
\hline $\mathrm{O}^{\mathrm{ii}}-\mathrm{Zn} 3-\mathrm{O}^{\mathrm{vi}}$ & $179.71(11)$ \\
\hline $\mathrm{O} 1^{\mathrm{ii}}-\mathrm{Zn} 3-\mathrm{O}^{\mathrm{vi}}$ & $90.3(1)$ \\
\hline 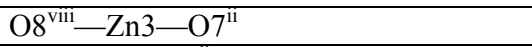 & $84.44(9)$ \\
\hline $\mathrm{O} 8-\mathrm{Zn} 3-\mathrm{O}^{\mathrm{ii}}$ & $95.38(9)$ \\
\hline $\mathrm{O} 1^{\mathrm{ii}}-\mathrm{Zn} 3-\mathrm{O}^{\mathrm{ii}}$ & $104.75(8)$ \\
\hline $\mathrm{O} 1^{\mathrm{Vi}}-\mathrm{Zn} 3-\mathrm{O}^{\mathrm{ii}}$ & $75.46(8)$ \\
\hline $\mathrm{O} 8^{\mathrm{viii}}-\mathrm{Zn} 3-\mathrm{O}^{\mathrm{vi}}$ & $95.38(9)$ \\
\hline $\mathrm{O} 8-\mathrm{Zn} 3-\mathrm{O}^{\mathrm{vi}}$ & $84.44(9)$ \\
\hline $\mathrm{O}^{\mathrm{ii}}-\mathrm{Zn} 3-\mathrm{O}^{\mathrm{vi}}$ & $75.46(8)$ \\
\hline $\mathrm{O} 1^{\mathrm{vi}}-\mathrm{Zn} 3-\mathrm{O} 7^{\mathrm{vi}}$ & $104.76(8)$ \\
\hline \multicolumn{2}{|c|}{ Distances Zn(4) - O (̊̊) } \\
\hline $\mathrm{Zn} 4-\mathrm{O} 13$ & $2.013(3)$ \\
\hline $\mathrm{Zn} 4-\mathrm{O} 13^{\mathrm{ix}}$ & $2.013(3)$ \\
\hline $\mathrm{Zn} 4-\mathrm{O} 4^{\text {iii }}$ & $2.079(2)$ \\
\hline $\mathrm{Zn} 4-\mathrm{O} 4^{\mathrm{x}}$ & $2.079(2)$ \\
\hline $\mathrm{Zn} 4-\mathrm{O} 11^{\mathrm{iii}}$ & $2.451(2)$ \\
\hline $\mathrm{Zn} 4-\mathrm{O} 11^{\mathrm{x}}$ & $2.451(2)$ \\
\hline Distance moyenne $<\mathrm{Zn}(4)-\mathrm{O}>$ & 2.181 \\
\hline \multicolumn{2}{|l|}{ Angles O - Zn(4) - O ( $\left.{ }^{\circ}\right)$} \\
\hline $\mathrm{O} 13-\mathrm{Zn} 4-\mathrm{O} 13^{\mathrm{ix}}$ & $94.1(1)$ \\
\hline $\mathrm{O} 13-\mathrm{Zn} 4-\mathrm{O} 4^{\text {iii }}$ & $93.75(9)$ \\
\hline $\mathrm{O} 13^{\mathrm{ix}}-\mathrm{Zn} 4-\mathrm{O} 4^{\mathrm{x}}$ & $93.75(9)$ \\
\hline $\mathrm{O} 4^{\text {iii }}-\mathrm{Zn} 4-\mathrm{O}^{\mathrm{x}}$ & $81.9(1)$ \\
\hline $\mathrm{O} 13-\mathrm{Zn} 4-\mathrm{O} 11^{\mathrm{iii}}$ & $85.11(9)$ \\
\hline $\mathrm{O} 13^{\mathrm{ix}}-\mathrm{Zn} 4-\mathrm{O} 11^{\mathrm{iii}}$ & $111.7(1)$ \\
\hline $\mathrm{O} 4^{\mathrm{iii}}-\mathrm{Zn} 4-\mathrm{O} 11^{\mathrm{iii}}$ & $82.10(8)$ \\
\hline $\mathrm{O} 4^{\mathrm{x}}-\mathrm{Zn} 4-\mathrm{O} 11^{\mathrm{iii}}$ & $79.78(8)$ \\
\hline $\mathrm{O} 13^{\mathrm{ix}}-\mathrm{Zn} 4-\mathrm{O} 4^{\mathrm{iii}}$ & $164.70(10)$ \\
\hline $\mathrm{O} 13-\mathrm{Zn} 4-\mathrm{O} 11^{\mathrm{x}}$ & $111.7(1)$ \\
\hline $\mathrm{O} 13^{\mathrm{ix}}-\mathrm{Zn} 4-\mathrm{O} 11^{\mathrm{x}}$ & $85.11(9)$ \\
\hline $\mathrm{O} 4^{\mathrm{iii}}-\mathrm{Zn} 4-\mathrm{O} 11^{\mathrm{x}}$ & $79.78(8)$ \\
\hline $\mathrm{O} 4^{\mathrm{x}}-\mathrm{Zn} 4-\mathrm{O} 11^{\mathrm{x}}$ & $82.10(8)$ \\
\hline \multicolumn{2}{|l|}{ Distances P(1) - O (̊) } \\
\hline $\mathrm{P} 1-\mathrm{O} 13$ & $1.494(2)$ \\
\hline $\mathrm{P} 1-\mathrm{O} 4$ & $1.517(2)$ \\
\hline $\mathrm{P} 1-\mathrm{O} 9$ & $1.535(2)$ \\
\hline
\end{tabular}

\begin{tabular}{|c|c|}
\hline $\mathrm{P} 1-\mathrm{O} 12$ & $1.600(2)$ \\
\hline Distance moyenne $<\mathrm{P}(1)-\mathrm{O}>$ & 1.5365 \\
\hline \multicolumn{2}{|c|}{ Angles $\mathrm{O}$ - P(1) - O $\left(^{\circ}\right)$} \\
\hline $\mathrm{O} 13-\mathrm{P} 1-\mathrm{O} 4$ & $111.8(1)$ \\
\hline O13-P1-O9 & $113.2(1)$ \\
\hline $\mathrm{O} 4-\mathrm{P} 1-\mathrm{O} 9$ & $111.9(1)$ \\
\hline $\mathrm{O} 13-\mathrm{P} 1-\mathrm{O} 12$ & $113.4(1)$ \\
\hline $\mathrm{O} 4-\mathrm{P} 1-\mathrm{O} 12$ & $107.1(1)$ \\
\hline $\mathrm{O} 9-\mathrm{P} 1-\mathrm{O} 12$ & $99.0(1)$ \\
\hline \multicolumn{2}{|c|}{ Distances P(2) - O $(\AA)$} \\
\hline $\mathrm{P} 2-\mathrm{O} 8$ & $1.490(2)$ \\
\hline $\mathrm{P} 2-\mathrm{O} 10$ & $1.514(2)$ \\
\hline $\mathrm{P} 2-\mathrm{O} 1$ & $1.526(2)$ \\
\hline $\mathrm{P} 2-\mathrm{O} 14$ & $1.618(2)$ \\
\hline Distance moyenne $<\mathrm{P}(2)-\mathrm{O}>$ & 1.537 \\
\hline \multicolumn{2}{|c|}{ Angles O - P(2) - O ( $\left.{ }^{\circ}\right)$} \\
\hline $\mathrm{O} 8-\mathrm{P} 2-\mathrm{O} 10$ & $113.9(1)$ \\
\hline $\mathrm{O} 8-\mathrm{P} 2-\mathrm{O} 1$ & $112.2(1)$ \\
\hline $\mathrm{O} 10-\mathrm{P} 2-\mathrm{O} 1$ & $112.7(1)$ \\
\hline $\mathrm{O} 8-\mathrm{P} 2-\mathrm{O} 14$ & $108.1(1)$ \\
\hline $\mathrm{O} 10-\mathrm{P} 2-\mathrm{O} 14$ & $107.2(1)$ \\
\hline $\mathrm{O} 1-\mathrm{P} 2-\mathrm{O} 14$ & $102.1(1)$ \\
\hline \multicolumn{2}{|c|}{ Distances P(3) - O $(\AA)$} \\
\hline $\mathrm{P} 3-\mathrm{O} 11$ & $1.511(2)$ \\
\hline $\mathrm{P} 3-\mathrm{O} 3$ & $1.528(2)$ \\
\hline $\mathrm{P} 3-\mathrm{O} 5$ & $1.541(2)$ \\
\hline $\mathrm{P} 3-\mathrm{O} 14$ & $1.583(2)$ \\
\hline Distance moyenne $<\mathrm{P}(3)-\mathrm{O}>$ & 1.54075 \\
\hline \multicolumn{2}{|c|}{ Angles O - P(3) - O $\left({ }^{\circ}\right)$} \\
\hline $\mathrm{O} 11-\mathrm{P} 3-\mathrm{O} 3$ & $112.3(1)$ \\
\hline $\mathrm{O} 11-\mathrm{P} 3-\mathrm{O} 5$ & $113.1(1)$ \\
\hline $\mathrm{O} 3-\mathrm{P} 3-\mathrm{O} 5$ & $110.9(1)$ \\
\hline $\mathrm{O} 11-\mathrm{P} 3-\mathrm{O} 14$ & $105.6(1)$ \\
\hline $\mathrm{O} 3-\mathrm{P} 3-\mathrm{O} 14$ & $108.0(1)$ \\
\hline $\mathrm{O} 5-\mathrm{P} 3-\mathrm{O} 14$ & $106.7(1)$ \\
\hline \multicolumn{2}{|c|}{ Distances P(4) - O (Á) } \\
\hline $\mathrm{P} 4-\mathrm{O} 6$ & $1.494(2)$ \\
\hline $\mathrm{P} 4-\mathrm{O} 7$ & $1.514(2)$ \\
\hline $\mathrm{P} 4-\mathrm{O} 2$ & $1.533(2)$ \\
\hline $\mathrm{P} 4-\mathrm{O} 12$ & $1.604(2)$ \\
\hline Distance moyenne $<\mathrm{P}(4)-\mathrm{O}>$ & 1.53625 \\
\hline \multicolumn{2}{|c|}{ Angles O - P(4) - O $\left(^{\circ}\right)$} \\
\hline $\mathrm{O} 6-\mathrm{P} 4-\mathrm{O} 7$ & $115.5(1)$ \\
\hline $\mathrm{O} 6-\mathrm{P} 4-\mathrm{O} 2$ & $112.70(1)$ \\
\hline $\mathrm{O} 7-\mathrm{P} 4-\mathrm{O} 2$ & $112.0(1)$ \\
\hline $\mathrm{O} 6-\mathrm{P} 4-\mathrm{O} 12$ & $106.7(1)$ \\
\hline $\mathrm{O} 7-\mathrm{P} 4-\mathrm{O} 12$ & $104.9(1)$ \\
\hline $\mathrm{O} 2-\mathrm{P} 4-\mathrm{O} 12$ & $104.3(1)$ \\
\hline
\end{tabular}

Code de symétrie : (i) $x,-y+1,-z+1$; (ii) $x-1 / 2, y-1 / 2, z$; (iii) $x-1 / 2,-y+3 / 2,-z+1$; (iv) $x+1 / 2, y-1 / 2, z$; (v) $-x+1, y,-$ $z+3 / 2$; (vi) $-x+1 / 2, y-1 / 2,-z+3 / 2$; (vii) $-x+1 / 2, y+1 / 2$, $z+3 / 2$; (viii) $-x, y,-z+3 / 2$; (ix) $x,-y+2,-z+1$; (x) $x-1 / 2$, $y+1 / 2, z$; (xi) $x+1 / 2, y+1 / 2, z$; (xii) $x+1 / 2,-y+3 / 2,-z+1$ 


\section{Conclusion}

Le nouveau pyrophosphate métallique mixte : $\mathrm{Cd}_{0.74} \mathrm{Zn}_{1.76} \mathrm{Fe}\left(\mathrm{P}_{2} \mathrm{O}_{7}\right)_{2}$ a été synthétisé par réaction de diffusion à l'état solide, et a été caractérisé par diffraction de rayons X sur monocristal. Ce nouveau pyrophosphate cristallise dans le système orthorhombique avec le groupe d'espace $\mathrm{C} 222_{1}$ et les paramètres de maille :

$a=8,4761$ (1) $\AA, b=9,7581$ (1) $\AA$, c $=24,0557$ (3) $\AA$, $\mathrm{V}=1989.66$ (4), $\mathrm{Z}=8$. La structure de ce nouveau composé est formée par des polyèdres métalliques déformés : quatre octaèdres $\mathrm{MO}_{6}(\mathrm{M}=\mathrm{Zn}, \mathrm{Fe}, \mathrm{Cd}(1) / \mathrm{Zn}(1))$, et une pyramide à base carrée $(\mathrm{Cd}(2) / \mathrm{Zn}(2)) \mathrm{O}_{5}$, formant des feuillets ondulés parallèles interconnectés par les groupements isolés $\mathrm{P}_{2} \mathrm{O}_{7}$ le long de (010).

\section{Références}

1. C. Bouzidi, W. Frigui, and M. F. Zid, Acta Cryst. E,71,1,69-72, (2015).

2. S. Loridant, I. C. Marcu, G. Bergeret,J. M. M. Millet, Phys. Chem. Chem. Phys, 5, 20, 4384-4389, (2003).

3. R. J. Xie , A. F. MacKenzie,Soil Sci. Soc. Am. J, 54,1,71-77, (1990).

4. D. H. Lee, S. Y. Kim,J. I. Hong, Angew. Chemie Int. Ed,43,36,4777-4780, (2004).

5. A.Gupta,N.B.Amin,A.Besarab,S.E.Vogel, G.W.

Divine, J.yee,J.V.Anandan,Kidney Int,55,5, 18911898, (1999).

6. S. Navas-Carretero, A. M. Pérez-Granados, B. Sarriá,M. P. Vaquero,Nutrition,25, 1,20-24, (2009).

7. Y. Zhang, A. C. Marschilok, E. S. Takeuchi, K. J. Takeuchi, MRS Adv,2,7,395-400, (2017).

8. A. Bolarinwa, U. Gbureck, P. Purnell, M. Bold,L. M. Grover, Adv. Appl. Ceram,109, 5, 291-295, (2010).

9. I. R. Orriss, T. R. Arnett, and R. G. G. Russell, Curr. Opin. Pharmacol, 28,7-68, (2016).

10. B. Lin, S. Zhang, and C. Deng, J. Mater. Chem. $A, 4,7,2550-2559,(2016)$.

11. C. Deng, S. Zhang,B. Zhao,Energy Storage Mater,4,71-78, (2016).

12. C. Masquelier, F. D'Yvoire, E. Bretey, P. Berthet,C. Peytour-Chansac, Solid State Ionics,67,3-4,183-189, (1994).

13. G. S. Gopalakrishna, B. H. Doreswamy, M . J. Mahesh, M. Mahendra, M.A. Sridhar, J. S.Prasad, K. G.Ashamanjari , Bull. Mater. Sci.,28,1,1-7, (2005).

14. Ģ. Vītiņš, Z. Kaņepe, A. Vītiņš, J. Ronis, A. Dindūne, A. Lūsis, J. Solid State Electrochem, 4,3,146-152, (2000).

15. A. Elmarzouki, A. Boukhari, E. M. Holt, A. Berrada, J. Alloys Compd,227,2, 25-130, (1995).

16. A. Boutfessi, A. Boukhari, E. M. Holt, Acta Cryst. Sect.C51,3,346-348, (1995).

17. A. Boutfessi, A. Boukhari, E. M. Holt, Acta Cryst. Sect.C52, 1594-1597, (1996).

18. A. Boutfessi, A. Boukhari, E. M. Holt, Acta Cryst. Sect.C52, 1597-1599, (1996).

19. K. H. Lii, P. F. Shih, and T. M. Chen, Inorg. Chem,32,20,4373-4377, (1993).

20. G. M. Sheldrick, Acta Cryst. Sect. A64, 1,112-122,
(2008).

21. B.Malaman, M.Ijjaali , R.Gerardin , G.Venturini, C.Gleitzer,Eur. J. Solid. State. Inorg . Chem,29, 1269-1284, (1992).

22. S.Boudin, A. Grandin,Ph. Labbe', D. Grebille, N.Nguyen, A. Ducouret, B. Raveau, J. Solid State Chem.121, 291-300, (1996)

23. A.ELBelghitti,A.Boukhari,M.E.Holt, Acta Cryst, C50,1648-1650, (1994).

24. A.Bronova, R.Glaum,C.Litterscheid. Acta Cryst, E69, i26, (2013). 